\title{
THE UNIVALENCE OF AN INTEGRAL OPERATOR
}

\author{
VIRGIL PESCAR
}

ABSTRACT. For analytic functions $f$ in the open unit disk $\mathcal{U}$, an integral operator $E_{\alpha, \beta}$ is defined. In this paper we derive univalence conditions of the integral operator $E_{\alpha, \beta}$.

Key Words: Analytic functions, Integral operator, Univalence.

2000 Mathematics Subject Classification: 30C45.

\section{INTRODUCTION}

Let $\mathcal{A}$ be the class of functions $f$ which are analytic in the open unit disk $\mathcal{U}=$ $\{z \in \mathbb{C}:|z|<1\}$, with $f(0)=f^{\prime}(0)-1=0$. Let $\mathcal{S}$ denote the subclass of $\mathcal{A}$ consisting of the functions $f \in \mathcal{A}$, which are univalent in $\mathcal{U}$. We denote by $\mathcal{P}$ the class of functions $p$ which are analytic in $\mathcal{U}, p(0)=1$ and $\operatorname{Re} p(z)>0$, for all $z \in \mathcal{U}$.

In this work, we define a new integral operator, which is given by

$$
E_{\alpha, \beta}(z)=\int_{0}^{z}\left(\frac{f(u)}{u}\right)^{\alpha}(g(u))^{\beta} d u,
$$

for $\alpha, \beta$ be complex numbers, $f \in \mathcal{A}$ and $g \in \mathcal{P}$.

From (1), for $\beta=0, \alpha$ be a complex number, $f \in \mathcal{A}$, we have the integral operator Kim-Merkes [2],

$$
I_{\alpha}(z)=\int_{0}^{z}\left(\frac{f(u)}{u}\right)^{\alpha} d u .
$$

For $\alpha=0, \beta$ be a complex number and $g \in \mathcal{P}$, we obtain the integral operator, which is defined by

$$
G_{\beta}(z)=\int_{0}^{z}(g(u))^{\beta} d u .
$$




\section{Preliminary Results}

Lemma 1. ([1]). If the function $f$ is analytic in $\mathcal{U}$ and

$$
\left(1-|z|^{2}\right)\left|\frac{z f^{\prime \prime}(z)}{f^{\prime}(z)}\right| \leq 1,
$$

for all $z \in \mathcal{U}$, then the function $f$ is univalent in $\mathcal{U}$.

Lemma 2. (Schwarz [3]). Let $f$ be the function regular in the disk

$\mathcal{U}_{R}=\{z \in \mathbb{C}:|z|<R\}$ with $|f(z)|<M, M$ fixed. If $f$ has in $z=0$ one zero with multiply $\geq m$, then

$$
|f(z)| \leq \frac{M}{R^{m}}|z|^{m},\left(z \in \mathcal{U}_{R}\right),
$$

the equality (in the inequality (5) for $z \neq 0$ ) can hold only if

$$
f(z)=e^{i \theta} \frac{M}{R^{m}} z^{m},
$$

where $\theta$ is constant.

\section{MAIN RESUlts}

Theorem 1. Let $\alpha \beta$ be complex numbers, $M_{1}, M_{2}$ positive real numbers and the functions $f \in \mathcal{A}, f(z)=z+a_{2} z^{2}+a_{3} z^{3}+\ldots$ and $g \in \mathcal{P}$, $g(z)=1+b_{1} z+b_{2} z^{2}+\ldots$

If

$$
\begin{gathered}
\left|\frac{z f^{\prime}(z)}{f(z)}-1\right| \leq M_{1}, \quad(z \in \mathcal{U}), \\
\left|\frac{z g^{\prime}(z)}{g(z)}\right| \leq M_{2}, \quad(z \in \mathcal{U}),
\end{gathered}
$$

and

$$
|\alpha| M_{1}+|\beta| M_{2} \leq \frac{3 \sqrt{3}}{2},
$$

then the function

$$
E_{\alpha, \beta}(z)=\int_{0}^{z}\left(\frac{f(u)}{u}\right)^{\alpha}(g(u))^{\beta} d u
$$

is in the class $\mathcal{S}$. 
Proof. The function $E_{\alpha, \beta}(z)$ is regular in $\mathcal{U}$ and $E_{\alpha, \beta}(0)=E_{\alpha, \beta}^{\prime}(0)-1=0$. We have:

$$
\frac{z E_{\alpha, \beta}^{\prime \prime}(z)}{E_{\alpha, \beta}^{\prime}(z)}=\alpha\left(\frac{z f^{\prime}(z)}{f(z)}-1\right)+\beta \frac{z g^{\prime}(z)}{g(z)}
$$

for all $z \in \mathcal{U}$.

From (10) we obtain:

$$
\left(1-|z|^{2}\right)\left|\frac{z E_{\alpha, \beta}^{\prime \prime}(z)}{E_{\alpha, \beta}^{\prime}(z)}\right| \leq\left(1-|z|^{2}\right)\left[|\alpha|\left|\frac{z f^{\prime}(z)}{f(z)}-1\right|+|\beta|\left|\frac{z g^{\prime}(z)}{g(z)}\right|\right],
$$

for all $z \in \mathcal{U}$. By Lemma 2, from (6) and (7) we get

$$
\begin{gathered}
\left|\frac{z f^{\prime}(z)}{f(z)}-1\right| \leq M_{1}|z|, \quad(z \in \mathcal{U}), \\
\left|\frac{z g^{\prime}(z)}{g(z)}\right| \leq M_{2}|z|, \quad(z \in \mathcal{U})
\end{gathered}
$$

and by (11) we have

$$
\left(1-|z|^{2}\right)\left|\frac{z E_{\alpha, \beta}^{\prime \prime}(z)}{E_{\alpha, \beta}^{\prime}(z)}\right| \leq\left(1-|z|^{2}\right)|z|\left(|\alpha| M_{1}+|\beta| M_{2}\right),
$$

for all $z \in \mathcal{U}$. Since

$$
\max _{|z| \leq 1}\left[\left(1-|z|^{2}\right)|z|\right]=\frac{2}{3 \sqrt{3}}
$$

by (14) and (8) we obtain

$$
\left(1-|z|^{2}\right)\left|\frac{z E_{\alpha, \beta}^{\prime \prime}(z)}{E_{\alpha, \beta}^{\prime}(z)}\right| \leq 1, \quad(z \in \mathcal{U})
$$

By Lemma 1, we obtain that the integral operator $E_{\alpha, \beta}$ belongs to the class $\mathcal{S}$.

Theorem 2. Let $\alpha, \beta$ be complex numbers and the functions $f \in \mathcal{S}, g \in \mathcal{P}$, $f(z)=z+a_{2} z^{2}+a_{3} z^{3}+\ldots, g(z)=1+b_{1} z+b_{2} z^{2}+\ldots$.

If

$$
2|\alpha|+|\beta| \leq \frac{1}{2},
$$

then the integral operator $E_{\alpha, \beta}$, defined by (1), is in the class $\mathcal{S}$. 
Proof. From (10) we obtain:

$$
\left(1-|z|^{2}\right)\left|\frac{z E_{\alpha, \beta}^{\prime \prime}(z)}{E_{\alpha, \beta}^{\prime}(z)}\right| \leq\left(1-|z|^{2}\right)\left[|\alpha|\left(\left|\frac{z f^{\prime}(z)}{f(z)}\right|+1\right)+|\beta|\left|\frac{z g^{\prime}(z)}{g(z)}\right|\right]
$$

for all $z \in \mathcal{U}$. Since $f \in \mathcal{S}, g \in \mathcal{P}$, we have:

$$
\begin{aligned}
& \left|\frac{z f^{\prime}(z)}{f(z)}\right| \leq \frac{1+|z|}{1-|z|}, \quad(z \in \mathcal{U}), \\
& \left|\frac{z g^{\prime}(z)}{g(z)}\right| \leq \frac{2|z|}{1-|z|^{2}}, \quad(z \in \mathcal{U})
\end{aligned}
$$

and hence, by (17) we get

$$
\left(1-|z|^{2}\right)\left|\frac{z E_{\alpha, \beta}^{\prime \prime}(z)}{E_{\alpha, \beta}^{\prime}(z)}\right| \leq 4|\alpha|+2|\beta|, \quad(z \in \mathcal{U}) .
$$

From (20), (16) we obtain

$$
\left(1-|z|^{2}\right)\left|\frac{z E_{\alpha, \beta}^{\prime \prime}(z)}{E_{\alpha, \beta}^{\prime}(z)}\right| \leq 1, \quad(z \in \mathcal{U})
$$

and by Lemma 1 , it results that $E_{\alpha, \beta} \in \mathcal{S}$.

\section{Corollaries}

Corollary 1. Let $\alpha$ be a complex number, $\alpha \neq 0$ and $f \in \mathcal{A}$, $f(z)=z+a_{2} z^{2}+a_{3} z^{3}+\ldots$ If

$$
\left|\frac{z f^{\prime}(z)}{f(z)}-1\right| \leq \frac{3 \sqrt{3}}{2|\alpha|}, \quad(z \in \mathcal{U}),
$$

then the integral operator $I_{\alpha}$, defined by (2), is in the class $\mathcal{S}$.

Proof. For $\beta=0$, from Theorem 1 we obtain Corollary 1 .

Corollary 2. Let $\beta$ be a complex number, $\beta \neq 0$ and $g \in \mathcal{P}$,

$$
g(z)=1+b_{1} z+b_{2} z^{2}+\ldots
$$

If

$$
\left|\frac{z g^{\prime}(z)}{g(z)}\right| \leq \frac{3 \sqrt{3}}{2|\beta|}, \quad(z \in \mathcal{U}),
$$

then the integral operator $G_{\beta}$ defined by (3), belongs the class $\mathcal{S}$. 
Corollary 3. Let $\alpha$ be a complex number and the function $f \in \mathcal{S}$, $f(z)=z+a_{2} z^{2}+a_{3} z^{3}+\ldots$

If

$$
|\alpha| \leq \frac{1}{4},
$$

then the integral operator $I_{\alpha}$ defined in (2), is in the class $\mathcal{S}$.

Proof. We take $\beta=0$ in Theorem 2, we obtain the Corollary 3.

Corollary 4. Let $\beta$ be a complex number and the function $g \in \mathcal{P}$, $g(z)=1+b_{1} z+b_{2} z^{2}+\ldots$

If

$$
|\beta| \leq \frac{1}{2},
$$

then the integral operator $G_{\beta}$ defined in (3), is in the class $\mathcal{S}$.

Proof. We take $\alpha=0$ in Theorem 2 .

\section{REFERENCES}

[1] Becker, J., Löwnersche Differentialgleichung Und Quasikonform

Fortsetzbare Schlichte Functionen, J. Reine Angew. Math. , 255 (1972), 23-43.

[2] Kim, Y. J. , E. P. Merkes, On an Integral of Powers of a Spirallike Function, Kyungpook Math. J., 12 (1972), 249-253.

[3] Mayer, O., The Functions Theory of One Variable Complex, Bucureşti, 1981.

Virgil Pescar

Department of Mathematics

"Transilvania" University of Braşov

500091 Braşov, Romania

email:virgilpescar@unitbv.ro 